Man and Nature

MAN AND NATURE

L'homme et la nature

L'HOMME ET LA NATURE

\title{
Le concept de souveraineté du peuple : de Rousseau à la Déclaration des droits de '93
}

\section{Josiane Boulad-Ayoub}

Volume 10, 1991

URI : https://id.erudit.org/iderudit/1012619ar

DOI : https://doi.org/10.7202/1012619ar

Aller au sommaire du numéro

Éditeur(s)

Canadian Society for Eighteenth-Century Studies / Société canadienne d'étude du dix-huitième siècle

ISSN

0824-3298 (imprimé)

1927-8810 (numérique)

Découvrir la revue

Citer cet article

Boulad-Ayoub, J. (1991). Le concept de souveraineté du peuple : de Rousseau à la Déclaration des droits de '93. Man and Nature / L'homme et la nature, 10, 13-30. https://doi.org/10.7202/1012619ar

Copyright (c) Canadian Society for Eighteenth-Century Studies / Sociéte canadienne d'étude du dix-huitième siècle, 1991
Ce document est protégé par la loi sur le droit d'auteur. L'utilisation des services d'Érudit (y compris la reproduction) est assujettie à sa politique d'utilisation que vous pouvez consulter en ligne.

https://apropos.erudit.org/fr/usagers/politique-dutilisation/ 


\section{Le concept de souveraineté du peuple: de Rousseau à la Déclaration des droits de ' 93}

Vous verriez Jean-Jacques Rousseau... le précurseur de cette grande Révolution; vous vous souviendriez qu'il vous apprenait à former des hommes pour la liberté lorsque vous étiez à la veille de faire des Français un peuple libre...Le Contrat Social a été pour vous la charte dans laquelle vous avez retrouvé les droits usurpés sur la nation, et surtout le droit imprescriptible de souveraineté. A.M. d'Eymar, Motion relative à J.-J. Rousseau, 29 décembre 1790.

\section{'LE DÉPUTÉ DE L'HUMANITÉ' ${ }^{1}$}

La souveraineté du peuple, le primat de la volonté générale sur les volontés particulières, l'égalité des droits, les concepts éminents de la doctrine jacobine telle que la prônent les orateurs révolutionnaires à la Convention, et en particulier leur chef de file, Robespierre, s'appuient sur des postulats anthropologiques aux conséquences sociales, hérités du rousseauisme: la nature a créé l'homme bon, libre, et perfectible, la vie sociale l'a corrompu, sa réforme passe par la réforme des institutions sociales et le développement de ses facultés naturelles par l'éducation, au sens large, et l'instruction. ${ }^{2}$ Réformer la société et ses lois nécessite donc pour Robespierre, tel qu'il comprend Rousseau, d'une part, de donner le primat à la souveraineté du peuple, d'autre part de former les moeurs qui sont à la base de la société civile et des pratiques de ses membres. Le politique appelle l'éthique et, réciproquement, les impératifs d'une religion déiste ainsi que d'une morale laïque cimenteront $l^{\prime}$ harmonie des rapports sociaux entre citoyens, entre les hommes vertueux que sont les membres de la nouvelle République, car la vertu est naturelle au peuple.

On exaltera donc l'amour de la vertu, attribut essentiel du peuple aussi bien que du gouvernement, et l'amour de la patrie. La vertu publique étant comme le rappelle Robespierre à la Convention dans son 
important Discours du 17 pluviôse an II, 'sur les principes de morale politique qui doivent guider la Convention Nationale dans l'administration intérieure de la République:' 'le principe fondamental du gouvernement démocratique et populaire, c'est-à-dire le ressort essentiel qui le soutient et le fait mouvoir. ${ }^{3} \mathrm{Et}$ Robespierre poursuit en identifiant très classiquement la vertu publique à l'amour de la patrie et de ses lois. Or, comme, d'une part, le peuple n'a besoin que d'une vertu, s'aimer luimême puisqu'il est vertueux par essence, dit Robespierre en transposant ainsi à l'homme collectif, au peuple, la notion d'amour de soi tel que l'entend positivement Rousseau par rapport à l'homme individuel, et comme, d'autre part, 'l'essence de la République ou de la démocratie est l'égalité, il s'ensuit que l'amour de la patrie embrasse nécessairement l'égalité. ${ }^{4}$ Si l'âme de la République est la vertu ou l'amour de l'égalité, si l'âme du peuple est la vertu, alors le patriotisme, c'est-à-dire l'amour de son pays et de ses lois, n'est pas autre chose, conclut Robespierre, que la vertu dans tout son état et la nature humaine dans toute sa dignité.

De là, la nécessité de moyens efficaces pour que réussisse la mission éthique-politique de la Révolution placée par Robespierre sous l'égide de la sagesse et de la vertu. On comptera sur les institutions républicaines conçues, avant la lettre, comme des appareils culturels chargés de produire, de maintenir et de renouveler le flux d'énergie révolutionnaire. Aux 'institutions' qui reprennent par là leur sens d'éducation est ainsi dévolue une tâche idéologique précise: faire passer dans les moeurs des citoyens et du gouvernement ce qui est garanti par les lois. Celles-ci sont les images de la liberté, de l'égalité, de la fraternité qui doivent régner entre les hommes nouveaux mais aussi elles ne sont que cela, des images, et non cette liberté, cette égalité, cette fraternité dans leur concrétude même. La force des institutions sera de combler l'écart, mieux, d'apprendre au peuple à faire mouvement de la liberté formelle aux libertés réelles, de l'égalité discursive à la réalité quotidienne, de $l^{\prime}$ humanité de principes au droit au travail et aux secours matériels des indigents.

Saint-Just met en évidence tout au début de ses Institutions, leur signification éthico-politique: 'Les institutions, écrit-il, sont la garantie de la liberté publique, elles moralisent le gouvernement et l'état civil; elles répriment les jalousies qui produisent les factions, elles établissent la distinction délicate de la vérité et de l'hypocrisie, de l'innocence et du crime; elles assoient le règne de la justice. Sans institutions la force $d^{\prime}$ une république repose ou sur le mérite des fragiles mortels, ou sur des moyens précaires. ${ }^{5} \mathrm{Et}$, un peu plus loin, le 'préambule' situe l'objet des institutions aussitôt après avoir défini le rôle social que celles-ci exercent sous le double rapport du peuple et du gouvernement: 'Les institutions sont la garantie du gouvernement d'un peuple libre contre la corruption 
des moeurs, et la garantie du peuple et du citoyen contre la corruption du gouvernement. Les institutions ont pour objet de mettre l'union dans les familles, l'amitié parmi les citoyens, de mettre l'intérêt public à la place de tous les intérêts, d'étouffer les passions criminelles[...] de mettre dans ${ }^{1}$ es citoyens et dans les enfants même une résistance légale et facile à l'injustice, de forcer les magistrats et la jeunesse à la vertu, de donner le courage et la frugalité aux hommes, de les rendre justes et sensibles.... ${ }^{6}$

Ainsi au concept politique de la souveraineté du peuple correspondra, sur le plan moral, la supériorité des devoirs exigés par l'être collectif, la société, ainsi que l'affirmation du degré plus élevé de perfection que comporte la notion de l'intérêt général par rapport à l'intérêt privé. On s'efforcera de diriger les passions du coeur humain vers les objets utiles à la prospérité publique. On apprendra aux hommes, c'est le rôle principal de l'éducation, à élever leurs âmes par la pratique des vertus privées, l'honnêteté, la sincérité, le courage, et des vertus civiques qui en sont inséparables, le respect pour la profonde dignité de l'homme; l'observation des bonnes lois qui sont bonnes en autant qu'elles sont conformes $\grave{a}$ la nature, $c^{\prime}$ est-à-dire à la raison [et] qui établissent cette heureuse harmonie, ${ }^{8}$ entre intérêt privé et intérêt général. C'est le critère qui permet de les distinguer des mauvaises lois, celles qui troublent cette harmonie.

La société rendra conscient dans l'homme son sens inné de l'obligation morale, thèse qui se transformera aisément comme dans le Contrat en thèse religieuse. ${ }^{9} C^{\prime}$ est pourquoi, on excitera sa croyance dans les principes d'une religion, une religion dont le rôle politique auquel est sensible Robespierre, lecteur tout autant de Montesquieu que de Rousseau sur ce point, est de contribuer, tout en appuyant la morale, à l'équilibre de la société. On incitera, pareillement, à l'obéissance des commandements donnés à l'homme par un Dieu qui ne peut qu'être bon puisqu'il est, selon l'argumentation rousseauiste, l'auteur d'un univers de bonté. ${ }^{10}$ Le bonheur et la liberté des individus comme de la société dépendent de l'application de ces principes. Aussi bien le salut du peuple viendra du développement de ses facultés naturelles, morales et intellectuelles, que fortifie l'exercice de ses droits naturels reconnus et protégés par une Constitution adéquate et les institutions politiques correspondantes. La régénération de l'homme et du citoyen, la révolution politique et morale, telle est l'oeuvre grandiose à laquelle s'attelleront pour la fonder, tout ensemble, l'éducation nationale et les lois de la République. 'Cette Convention, déclarera Robespierre en rappelant les tâches de la Révolution, devra trois monuments à l'histoire: la constitution, le code des lois civiles, l'éducation publique. ${ }^{11}$ 


\section{LA VERTU ET LA CONSTITUTION}

La Constitution ${ }^{12}$ a pour mission de fixer les lois fondamentales de la Nation mais celles-ci devront découler des lois éternelles de la justice et de la raison. Les lois de la justice car elles respectent les droits de l'humanité alias le peuple, les lois de la raison, car elles vainquent les deux fléaux qui l'accablent, l'ignorance et le despotisme, et qu'elles révèlent par là leur caractère éternellement salvateur. Il s'agit de la raison naturelle et laïque de l'homme des Lumières, une raison synonyme du Progrès et de la Liberté, une force active en lutte contre les forces obscurantistes et réactionnaires. Les lois civiles doivent pour Robespierre tirer leur force des lois de la morale naturelle. Il renverse sur ce point la doctrine dominante des Lumières pour laquelle les fondements de l'obligation morale découlent au contraire des impératifs de la vie en société ${ }^{13}$ et, qui, par conséquent, rabat l'éthique sur le politique; celui-ci étant alors, en raison de l'immobilisme des structures qui l'articulent, le champ privilégié de la lutte idéologique des Philosophes et des réformes normatives qu'ils préconisent. Aussi la notion de vertu, qui joue un si grand rôle dans l'axiologie révolutionnaire, et celle de Robespierre en particulier, est-elle entendue par les Encyclopédistes d'abord comme une notion morale qui doit s'aligner sur le critère primordial de l'utilité ${ }^{14}$ et du bonheur. 'Rendre les hommes heureux par la vertu: voilà le grand problème que la morale doit se proposerde résoudre,' déclare d'Holbach dans le Système Social. ${ }^{15}$ Pour Robespierre, au contraire, qui pense sous des conditions politiques différentes, pour qui les transformations sociales appelées par les hommes des Lumières ont effectivement eu lieu grâce à l'action révolutionnaire du peuple $-c^{\prime}$ est ce qui du reste confère à ce dernier sa vertu, sa puissance et sa majesté -, le politique doit trouver sa justification dans la sorte de légitimation transcendentale que lui offrent les principes éternels de la morale et la religion. Et, par un second renversement, un chiasme s'opère sur le plan conceptuel, dicté cette fois par les nécessités pratiques de l'engineering sociétal, si l'on veut bien nous passer cette expression anachronique. La vertu et la félicité des hommes deviennent à ce compte, et la vertu, au premier chef, des notions politiques; et le problème du bonheur, le grand problème que doit résoudre l'homme politique et non le moraliste.

$L$ 'inscription de la vertu dans le domaine moral, chez les Philosophes, dans le domaine politique chez Robespierre, a également des conséquences sur le renouvellement de significations du concept qui lui est le plus voisin à l'intérieur du champ sémantique (nature $>><<$ raison $>><<$ homme $>><<$ vertu $>><<$ liberté $>><<$ égalité $>><<$ bonheur $>>$ $<<$ progrès $>><<$ société) où tous deux travaillent: il s'agit du concept d'humanité et de son doublet révolutionnaire, le concept de fraternité. 
La vertu entendue d'abord dans un sens moral apparente la pratique de la vertu au devoir d'humanité envers ses semblables; la notion d'humanité évinçant progressivement dans le koînon du moment la notion de fraternité et ses connotations qui apparaissent alors plus religieuses et chrétiennes que civiques. Chez Robespierre, en revanche, et à partir du déplacement de la notion de vertu sur le terrain politique, la pratique de la vertu pour être complète exige la solidarité-fraternité entre membres de la société, entre patriotes. ${ }^{16}$ Et l'un des objectifs de l'institution du culte de l'Être suprême et des Fêtes nationales glorifiant les concepts abstraits mis à l'honneur par la morale républicaine de la Vertu, vise expressément à ressouder les citoyens de diverses catégories sociales dans une même foi civile et, par là, comme s'écrie Robespierre, s'inspirant de Rousseau, à resserrer les doux noeuds de la fraternité universelle. ${ }^{17}$

Seules des lois humaines, d'un mot qui rassemble lois civiles et lois morales, réconciliant par et dans le sujet-agent qui fait ces lois et qui les met en oeuvre, autrement dit le peuple souverain, permettront de mettre en pratique les dispositions prévues par la loi, et, de manière concomitante, feront que la vertu cesse d'être un vain mot, limité aux bonnes intentions du langage moral. Ainsi l'idéalisme moral rousseauiste et la religiosité diffuse de sa pensée, combinés à l'héritage humaniste des Philosophes auxquels viennent s'ajouter les déterminations politiques de la conjoncture, tout cela fait, si l'on veut parler rapidement, que Robespierre, en réaliste et en homme d'action qu'il est, pose les fondements du droit dans le domaine d'une éthique humaniste mais qu'il conçoit réciproquement la vertu comme la pratique politique par excellence, celle dont dépend éminemment le fonctionnement optimal de la machine sociale.

Seules donc pour Robespierre les lois qui seront entés sur les principes éternels de la justice et de la raison publique comme sur les droits naturels et imprescriptibles qui ne font que les exprimer à travers l'histoire de toute association politique, permettront à ses yeux d'articuler concrètement, tel est le mot clé, les droits de l'homme à ceux du citoyen. Subordonnant l'intérêt particulier à l'intérêt général, puisqu'elles ne défendent que ce qui est nuisible à la société, qu'elles n'ordonnent que ce qui lui est utile, ${ }^{18}$ les lois garantissent par là même, par leur accord avec la volonté générale, la souveraineté et la dignité du peuple. À leur tour, de telles lois, peuvent être promulguées par l'Assemblée en tant que les députés agissent au nom du peuple et que les lois proposées visent à l'accomplissement de ses droits, à l'épanouissement de ses facultés naturelles en même temps qu'à le récompenser d'une action politique et nationale rigoureuse. On le voit bien avec les décrets de Ventôse qui donnent sans doute à la propriété privée un nouveau fondement juridique, mais qui lui sert en même temps, et surtout, 
d'assise morale. 'Si vous donnez des terres à tous les malheureux, déclare Saint-Just, si vous les ôtez à tous les scélérats, je reconnais que vous aurez fait une révolution. ${ }^{19}$

\section{LES JEUX DE LA MIMÊSIS DANS LA DÉCLARATION DE '93}

Le projet de Déclaration des Droits que Robespierre présente le 24 avril 1793 devant la Convention, constitue le prémices d'une véritable démocratie sociale. ${ }^{20}$ Elle a pour ambition de proclamer à la face de l'univers et sous les yeux du législateur immortel (Dieu ou la Nature) les droits sacrés et inaliénables de l'homme et du citoyen. L'ensemble de ces droits et leurs définitions forment le cadre théorique si l'on peut dire, à l'intérieur duquel devrait être élaboré le code des lois civiles, l'armature de la Constitution. Fidèle à son principe que le gouvernement, c'est-à-dire l'exécutif, est subordonné au souverain et doit toujours lui rendre compte s'il ne veut pas le trahir, Robespierre rend claires dans le solennel Préambule, les finalités profondes qui orientent l'exposé des droits du peuple, des droits de l'humanité, droits constitutifs de la Déclaration. Le corps de ladite Déclaration gravite toute entière autour du concept de souveraineté du peuple, on le verra dans un moment, et a pour charge, par conséquent, de détailler et d'expliciter les droits politiques qui en découlent.

Mais faire ainsi, c'est faire encore et en même temps oeuvre d'éducateur, puisque selon les termes mêmes de Robespierre, 'partout où les lois sont fondées sur ces principes [l'accord de l'intérêt privé avec l'intérêt général, comme il le dit un peu plus haut au cours du même paragraphe], elles sont elles-mêmes l'éducation publique. ${ }^{\prime 21}$ Les prolégomènes de la Déclaration, pour leur part, tout en affirmant le fondement transcendental des lois et des droits humains dans les lois éternelles de la justice et de la raison, développent en même temps les devoirs, ${ }^{22}$ pourrait-on dire, de $l^{\prime}$ homme et du citoyen, tenant ainsi lieu de contrepartie morale et individuelle aux droits politiques et collectifs du peuple.

C'est que Robespierre avec tout son siècle, et surtout avec Rousseau, joint aux préoccupations sociales qui l'habitent, au problème politique qu'il considère le plus important, établir la souveraineté du peuple et en garantir son effectuation, un souci didactique lequel converge avec son projet éthique jusqu'à se confondre avec lui. Ainsi verra-t-il dans le plan $\mathrm{d}^{\prime}$ éducation nationale ${ }^{23}$ rédigé par Le Peletier de Saint-Fargeau, un rousseauiste de la première heure comme la plupart des membres du Comité d'Instruction Publique, projet que Robespierre présente à la Convention le 13 juillet 1793 , la panacée universelle qui devrait régler, par la propagation des lumières, par la formation du coeur et de l'esprit, ${ }^{24}$ 
les questions sociales et économiques. De même prend-il soin dans le Préambule d'instruire ses concitoyens de la façon dont ils doivent user de la Déclaration pour que les buts que celle-ci est censée remplir restent toujours vivants. Robespierre, en même temps que l'énoncé des buts de la Déclaration, y convie en effet chaque citoyen à se livrer à l'activité critique la plus inlassable; il fera comparaître devant le tribunal de la raison politique, la pratique du gouvernement avec les finalités de la société. C'est en ceci que consistera pour l'essentiel la vertu républicaine, l'autonomie morale et politique du nouvel homme de l'homme. L'exercice de la vertu devient alors, tel est l'enjeu politico-moral du second objectif explicite de la Déclaration, les gages du bonheur et de la liberté que le peuple trouvera dans l'établissement des lois. De la même manière, le devoir moral du magistrat, en tant que délegué du peuple, sera de mesurer à ce qui forme les bases de sa liberté et de son bonheur, la règle d'application des lois, et celui du législateur, de délimiter l'objet de sa mission; celle-ci étant de venger les injures du peuple et de lui rendre toute sa dignité. ${ }^{25}$ Les nouvelles Institutions sociales devant aider à fortifier la vertu républicaine par l'implantation concrète des acquis théoriques et pratiques de la Révolutioin démocratique. Elles préviendront le peuple de se corrompre, et, réciproquement, les lois et le gouvernement de pérécliter.

La vertu, l'un des maîtres mots des Lumières, le premier en tous cas dans l'action politique et morale ${ }^{26}$ de Robespierre, est, comme il le dit souvent, l'âme de la République. ${ }^{27}$ Il la définit, à la suite de Montesquieu, comme l'amour de la patrie et de l'égalité, ${ }^{28}$ avec Rousseau, le dévouement magnanime qui confond tous les intérêts privés dans l'intérêt général. ${ }^{29}$ Le culte humaniste de la vertu, et partant, de la patrie et du peuple, vertueux par essence, renferme toute la doctrine morale de Robespierre tout de même que la croyance en l'Être Suprême et dans l'immortalité de l'âme constitueront la base de sa théorie de la religion civile. Toutes deux, la morale de la vertu et la religion de l'Être suprême, ont une fonction nettement idéologique. Robespierre voit dans la vertu, et l'on s'apercevra tantôt qu'il fera fond de la même manière sur le culte de l'Être suprême ainsi que sur l'institution des Fêtes Nationales pour réaliser ses objectifs éthico-politiques, la force capable par excellence de resserrer les liens sociaux. Seule la pratique de la vertu permet d'accorder le domaine de l'individuel et du social. Ce sera elle qui empêchera la voix de la volonté générale d'être étouffée par celle des intérêts particuliers. La liberté et l'égalité absolues sont sans doute chimériques, du moins elles restent lettre morte si la vertu, l'ensemble des devoirs sacrés et sublimes de tout homme envers la patrie et l'humanité, ne vient pas relayer activement dans le pacte social la proclamation des droits de tout citoyen et quel'on veille par son exercice à leur application. Saint-Just avait posé de même que 
'la liberté n'est aimable qu'autant qu'elle ramène à la simplicité par la force de la vertu. ${ }^{30}$

La vertu, à la fois au sens où l'entendaient l'Antiquité, (la figure de Brutus), Montesquieu et les Philosophes, est donc une force morale et psychologique, la force d'âme, le courage, mais aussi une force assimilable à la force d'une raison politiquement orientée, la raison publique, qui détermine le citoyen dans sa volonté de respecter les lois et de résister à ceux qui attentent à la liberté de la patrie, l'homme à compatir avec son semblable, son frère, devant les injures qui lui sont faites, à s'en sentir solidaire, bref à aimer avec la vertu, la vérité, la nature et la justice. Le mot de Saint-Just illustre bien cet engouement pour la vertu, si caractéristique de l'éthos révolutionnaire de l'époque: 'Une chose triomphe de tout sur la terre: $c^{\prime}$ est l'audace unie à la vertu. ${ }^{31}$ La vertu et l'audace de la raison, pratique et théorique sont bien les enjeux idéologiques clés qui gouvernent souterrainement le Préambule de cette Déclaration appelée dans les intentions de Robespierre à déployer légitimement la puissance et la bonté du peuple souverain.

La Déclaration doit permettre aux citoyens de comparer les actes $d u$ gouvernement avec le but de l'institution sociale tel que ce but est exprimé au long des articles; en particulier par l'article premier: 'Le but de toute association politique est le maintien des droits naturels et imprescriptibles de l'homme, et le développement de toutes ses facultés.' C'est dans ce jugement continuel de leur raison, dans cette évaluation constante que tous les citoyens sont enjoints de faire, que réside de manière active leur liberté. Si jamais le gouvernement déroge à l'intérêt général, alors le souverain a le droit et le devoir de cesser d'obéir aux lois. Le pacte social est violé et le peuple recourra à une légitime insurrection contre l'injustice et la tyrannie pour exercer sa souveraineté. C'est évidemment le concept éminent de la Déclaration, la souveraineté du peuple, qui est en jeu ici, et qui vient lester de son poids politique l'activité critique du citoyen. L'article XV qui le promulgue avec une netteté exemplaire: 'Le peuple est le souverain: le gouvernement est son ouvrage et sa propriété, les fonctionnaires publics sont ses commis,' n'est pas placé au hasard bien au centre des trente articles qui composent la Déclaration. Il agit comme son centre de gravité et détermine comme autant de ses fondements ou de ses conséquences, les articles qui le précèdent ou qui le suivent pout définir les droits de l'homme et du citoyen.

Le maintien des droits de l'homme et le développement de ses facultés naturelles: c'est l'article premier qui, énonçant de fait le but de la société républicaine, l'universalise normativement pour l'étendre de droit à toute association politique. Suivent la formulation de ces droits et les moyens prévus pour atteindre le bien, le bonheur universel par le développement des facultés. L'égalité des droits, en premier, la liberté 
- métaphysique et politique - et ses règles, la propriété déterminée par la loi. La loi, garante légitime de l'harmonie sociale, est décrite dans sa nature d'abord en regard de la société et de l'utilité publique, ensuite des droits de l'homme et du citoyen, pour être enfin définie sous l'autorité théorique du Contrat comme l'expression de la volonté générale. Les obligations de la société envers ses membres sont alors énumérées. Cette suite d'articles regroupent les principaux éléments de l'idéal social de Robespierre qui entend préciser comme autant de droits constitutionnels, la marque sur sa politique égalitariste aussi bien de l'Esprit des Lois, de l'Encyclopédie que du Contrat. Il s'agit du droit au travail que la société doit assurer à tous ses membres, les secours aux indigents que justifie la solidarité mutuelle devant régner entre citoyens, et dont les modalités seront délimités par la loi, l'instruction mise à portée de tous, et, partant, les progrès de la raison publique.

Tous ces droits qui se répartissent dans ce qu'on peut appeler la première série (les articles I à XI), détaillent, ou plutôt, explicitent en quoi consiste le but de toute institution sociale; les articles encadrent comme à l'avance le déroulement éventuel des actes du gouvernement. Leur contenu a pour enjeu de rendre le peuple conscient de sa souveraineté primordiale, de sa liberté essentielle; le Préambule faisant dynamiquement dépendre du peuple lui-même et de l'activité de sa raison, cette maîtrise et cette liberté. C'est alors littéralement que le peuple s'institue, quand il évalue le discours et les faits, les choses et les mots, quand il exerce son jugement, quand il fait respecter sa volonté et que sa raison devient à bon droit la raison publique, le garant de la liberté, de l'égalité, et de la félicité universelle.

La deuxième série des droits déroule les conséquences entraînées par le principe mis au coeur de la Déclaration, la souveraineté du peuple. Après la réunion par la première série des termes de la comparaison qui permet le jugement éclairé du souverain, Robespierre fait état des moyens dont celui dispose pour exercer sa liberté et sa puissance. Les articles XVI à XXX exposent l'équilibre à respecter entre les portions du peuple qui constituent la volonté générale; les diverses formes de l'égalité par rapport à la loi et telles que la concrétisent l'accessibilité aux fonctions publiques, le suffrage universel, les modes de rétribution des fonctions publiques. Le projet de Déclaration fait ensuite la longue énumération de toutes les formes légitimes de résistance à l'inégalité et à l'oppression, énumération qui culmine dans l'article XXVII: 'Quand le gouvernement opprime le peuple, l'insurrection du peuple entier et de chaque portion du peuple est le plus saint des devoirs.' Robespierre réaffirme enfin le principe voulant que la loi défende la liberté publique et individuelle contre les abus de l'autorité des gouvernants. Le dernier article pose à nouveau la souveraineté du peuple et le devoir essentiel de ses manda- 
taires; il consiste à rendre toujours compte devant le peuple de la gestion de la volonté du peuple. Aussi bien, telle est la conclusion, puisque la liberté et l'égalité sont l'apanage naturel (les droits naturels) du peuple souverain, celui-ci s'identifie au genre humain et les liens de fraternité débordent les frontières $d^{\prime}$ un pays, pour unir tous les peuples de la terre contre les tyrans du genre humain quels qu'ils soient. Le second but explicite de la Déclaration, but modélisateur s'il en est, commence d'être atteint dès la mise en vigueur de la Déclaration: le peuple aura toujours devant les yeux les bases de sa liberté et de son bonheur; le magistrat, la règle de ses devoirs; le législateur, $l^{\prime}$ objet de sa mission. ${ }^{32}$

Mais pour que le magistrat puisse tirer avec rectitude la règle de ses devoirs envers le peuple souverain, et pour que le législateur qui agit en son nom puisse élaborer les lois conformes à la dignité de ses droits, il leur faut connaître la nature de cet être collectif. La première chose que doit savoir le législateur, c'est que le peuple est bon. ${ }^{33}$ Robespierre fait de ce postulat l'article XXIX de son projet de Déclaration, soutenant par la bonté naturelle du peuple, le primat qu'il lui donne dans l'organisation politique. Il stipule: 'Toute institution qui ne suppose pas le peuple bon, et le magistrat corruptible, est vicieuse. ${ }^{34}$ La prééminence du souverain, du peuple par rapport au magistrat, autrement dit la thèse affirmant que la volonté générale associée au bien de l'humanité doit primer la volonté des individus et les passions particulières, s'éclaire mieux peut-être à la lecture du commentaire de Robespierre sur la question de savoir quel sera le meilleur précepteur des droits et des devoirs des citoyens, examen qu'il fait dans les Observations générales sur le projet d'instruction publique..., texte publié un peu plus tôt que celui du projet de Déclaration. Robespierre comparant la rectitude de la volonté générale aux flottements des volontés individuelles, accorde de ce fait la supériorité institutionnelle et législative au peuple-démiurge par lequel s'exprime cette volonté, et qui représente alors l'humanité: les volontés particulières ne faisant que reproduire les égoïsmes particuliers des divers individus, sont par là distinctes de l'intérêt commun. 'Le peuple, écrit Robespierre, vaut toujours mieux que les individus: or que sont les dépositaires de l'autorité publique, si ce ne sont des individus plus exposés que les autres à l'erreur? Le peuple veut toujours son propre bien; l'individu, l'homme puissant veut aussi le sien; mais le bien du peuple est l'intérêt de l'humanité, le bien particulier des hommes puissants, c'est l'intérêt de l'orgueil. ${ }^{35}$

Robespierre renvoie alors la Convention à la figure du Législateur telle qu'elle apparaît dans le Contrat.$^{36} \mathrm{Il}$ voudrait voir l'Assemblée agir à son image dans ses travaux législatifs, en philosophe éclairé et intrépide. Intrépide car, comme Rousseau le souligne lui-même, les qualités nécessaires au législateur sont telles qu'il faudrait des dieux pour donner des 
lois aux hommes. Sa mission, en effet, et il n'est pas interdit de penser que Robespierre aspirait à émuler le rôle du Législateur rousseauiste, est de changer la nature humaine au niveau des moeurs mais sans se substituer au peuple souverain en matière politique. De là sans doute, soit dit en passant, une des raisons de la confiance que met Robespierre dans le règne des lois et dans la diffusion de l'instruction pour hâter ces changements. Robespierre souhaiterait que les députés, ses collègues sachent s'inspirer du modèle que le plus éloquent de nos philosophes a tracé dans le Contrat. Il en grossit les traits, au bénéfice de ses lecteurs, - et de la cause à laquelle il s'identifie - qui apparentent les qualités du modèle à l'idéal vertueux de la morale civique et républicaine: le détachement à l'égard des passions, l'horreur de la tyrannie, l'amour de l'humanité, le respect pour le peuple, le souci constant de lui rendre ses droits et sa dignité. ${ }^{37}$

L'homme collectif, le peuple est donc bon, il recèle en lui la vertu, mais il est aussi libre, 'le souverain bien de tout être qui n'est pas abruti.' Robespierre définit cette propriété dans la Déclaration comme droit naturel et politique: 'la liberté est le pouvoir qui appartient à l'homme d'exercer à son gré toutes ses facultés. Elle a la justice pour règle, les droits d'autrui pour bornes, la nature pour principes, et la loi pour sauvegarde. ${ }^{38}$ Robespierre reprend ici presque textuellement la définition que donne Rousseau de la liberté dans le Contrat en lui adjoignant des précisions - les principes de la morale naturelle, la garantie des lois - qu'il emprunte à la conception de Montesquieu et à l'article Liberté de l'Encyclopédie où la liberté est faite par la loi. Rousseau écrit à l'intérieur d'une note qui résume elle-même un développement qu'il a trouvé dans l'ouvrage de d'Argenson, Considérations sur le gouvernement ancien et présent de la France: 'Dans la république, dit le marquis d'Argenson, chacun est parfaitement libre en ce qui ne nuit pas aux autres. Voilà la borne invariable; on ne peut la poser plus exactement. ${ }^{, 39}$

Les ennemis du peuple seront donc ceux qui attentent à cette propriété naturelle érigée immédiatement en droit politique, ${ }^{40}$ et qui, par conséquent, en menaçant la liberté du peuple tentent en même temps de l'avilir par la tyrannie ou tout autre forme de despotisme. Robespierre se souvient sans doute ici de Rousseau et des fortes paroles du Contrat: 'Renoncer à sa liberté, c'est renoncer à sa qualité d'homme, aux droits de l'humanité, même à ses devoirs [...] c'est ôter toute moralité à ses actions que d'ôter toute liberté à sa volonté..$^{41}$ La liberté est donc un état que l'homme détient par nature, que la société affermit par ses lois et par ses institutions, mais dont il a pour charge, comme homme et comme citoyen de maintenir activement. En vertu de cette conception, la défense de la liberté autorise, dicte même la violence, la terreur et la mort. Le 20 juillet 1789, Robespierre s'écriait à l'Assemblée, justifiant le 
rôle salvateur pour la République des violences populaires du 14 juillet: 'Eh messieurs, c'est à cette émeute que la Nation doit la liberté. ${ }^{42}$ Trois mois après le début de la Révolution, il plaçait déjà les faits et les effets de la liberté, les actes du peuple libre plus haut que le discours formel des Constituants: 'Que l'on ne nous parle pas tant de constitution; ce mot nous a trop endormis, nous a trop retenus dans la léthargie; cette constitution ne sera qu'un vain livre; et qu'importe qu'il soit fait quand on nous aura ravi notre liberté au berceau. ${ }^{, 43}$

La position de Robespierre est prise depuis longtemps. Sa critique du despotisme, ses idées sur la séparation des pouvoirs, sa théorie de la liberté doivent autant à la pensée de Montesquieu, de Diderot et de d'Holbach qu'à celle de Rousseau qu'il privilégie. Il ne s'en cache pas, mais elles ont été épurées au creuset de l'épreuve révolutionnaire. La lutte contre les ennemis de la liberté doit être conçue positivement comme une lutte pour la liberté, dynamiquement comme une recréation de l'homme, politiquement comme l'accomplissement de la volonté du peuple. Quand le gouvernement dégénère en tyrannie, c' est-à-dire lorsqu'il agit contre la volonté et contre l'intérêt de la société elle-même, alors le pacte politique est rompu, alors c'est le moment pour le peuple de se lever.

Développant une argumentation rousseauiste (elle-même répétant un développement-choc du Système de la nature de d'Holbach) qu'il avait déjà utilisé dans son discours qui requerrait la peine de mort pour Louis XVI, Robespierre soutient le raisonnement suivant. La nation, dans le cas où on a abusé de sa liberté, est en état de guerre contre le tyran, et elle rentre, à son égard, dans tous les droits de la nature. Ce n'est plus $\mathrm{d}^{\prime}$ après les lois qu'il a violées que le tyran doit être jugé, mais d'après les principes de la loi naturelle sous l'empire de laquelle il s'est replacé lui-même. Or que prescrit cette loi? Elle impose aux hommes un double devoir; le premier est celui de pourvoir à leur propre conservation, d'où résulte le droit de punir tous ceux qui attaquent leur liberté ou leur sûreté; le second est de secourir nos semblables opprimés, d'où dérive encore le droit de punir ceux qui les oppriment. ${ }^{44}$ L'intervention vigoureusement célèbre de Robespierre contre le veto absolu ou royal puis contre le veto suspensif que Barnave voulait introduire à l'époque de la Constituante, résume bien les principes à la base de son option démocratique et de la conceptioin de la liberté-égalité qu'elle entraîne. Ainsi se déclarant contre le veto royal, Robespierre affirme dans son discours à l'Assemblée: 'Celui qui dit qu'un homme a le droit de s'opposer à la loi dit que la volonté d'un seul est au-dessus de la volonté de tous. Il dit que la Nation n'est rien et qu'un seul homme est tout. $S^{\prime}$ il ajoute que ce droit appartient à celui qui est revêtu du pouvoir exécutif, il dit que l'homme établi par la Nation pour faire exécuter les volontés de la 
Nation a le droit de contrarier et d'enchaîner les volontés de la Nation. Il a créé un monstre inconcevable en morale et en politique, et ce monstre n'est autre que le Veto Royal. ${ }^{45}$ Quant au veto suspensif il y voit la paralysie du pouvoir législatif, c'est-à-dire de la Nation par le pouvoir exécutif et donc une attaque redoutable à la Liberté.

\section{LA FÉLICITÉ UNIVERSELLE...}

La Déclaration marque de façon solennelle le début du nouveau pacte social, l' instant heureux qui, comme disait Rousseau, $d^{\prime}$ un animal stupide et borné, fit un être intelligent et un homme. ${ }^{46}$ Plate-forme idéologique et politique des idéaux jacobins, elle devait rassembler autour d'elle et de la nouvelle Constitution qu'elle préface, toute la nation; les nouvelles institutions républicaines perpétuer cet instant et assurer sur les fondements des lois et la souveraineté du peuple, le règne civil de l'esprit de liberté et d'égalité. Cet esprit d'égalité n'étant point, comme l'écrit SaintJust, que l'homme puisse dire à l'homme, je suis aussi puissant que toi [...], l'esprit de l'égalité est que chaque individu soit une portion égale de la souveraineté, $c^{\prime}$ est-à-dire du tout. ${ }^{47}$ Les institutions de la République sont désignées pour établir, ainsi qu'on le dirait en un langage moderne qui sonne familièrement à nos oreilles, la dictature du peuple et la révolution culturelle comme nécessités objectives. Elles ont pour fonction de reconduire à chaque moment de la vie sociale, ce que la Révolution et le gouvernement révolutionnaire a fait gagner aux hommes bons et libres par nature: la souveraineté, la liberté politique, la liberté morale, l'égalité de convention, de droit et de fait, tous ces biens qui ont été substitués à l'injustice et à l'oppression de l'ancien régime. C'est aussi, et surtout, sur les institutions républicaines que reposent les tâches de lentement développer et de transformer, ce faisant, les déterminants physiques et moraux du système social, les moeurs, les comportements, les pratiques, d'un mot, l'habitus des citoyens qui en sont partie prenante.

Reconstruire les bases de l'édifice social selon les lois justes et bonnes de la raison et de la nature, regénérer les hommes dépravés jusqu'alors par les formes et les moeurs sociales auxquelles ils étaient assujetis est une tâche de longue haleine dont la Constitution marque l'aurore, mais ce n'est pas une tâche utopique. C'est une tâche patiente. Robespierre postule avec ses camarades révolutionnaires, avec tout son siècle, l'irrésistibilité du progrès, le caractère perfectible de l'homme, une perfectibilité qui s'avère solidaire, sur le plan éducatif, du progrès épistémique, technique et social. La vigilance et l'énergie ${ }^{48}$ révolutionnaires des citoyens, assistés par la toute puissance de la raison et tout à la fois de la vertu qui amplifient leurs facultés, permettront de lutter contre les ennemis 
de la société républicaine comme de faire sauter les demières barrières sur les voies conduisant à la félicité universelle. C'est alors que l'invincibilité de la Révolution se révélera, que le peuple sera définitivement vainqueur, que le monde tant désiré de la liberté, de l'égalité, de la justice et du bonheur seront désormais pour tous à portée de main. Dira Robespierre:

Que la France jadis illustre parmi les pays esclaves, éclipsant la gloire de tous les peuples libres qui ont existé, devienne le modèle des nations, l'effroi des oppresseurs, la consolation des opprimées, l'ornement de l'univers, et qu'en scellant notre ouvrage de notre sang, nous puissions voir au moins briller l'aurore de la félicité universelle. ${ }^{49}$

\section{JOSIANE BOULAD-AYOUB \\ Université du Quebéc à Montréal}

\section{Notes}

1 C'est ainsi que s'exprime Saint-Just en août 1790 ăăns une lettre adressée à Robespierre, de Blérancourt: 'Je ne vous connais pas, mais vous êtes un grand homme. Vous n'êtes point seulement le député d'une province, vous êtes celui de l'humanité.' in Saint-Just, Discours et rapports, éd. A. Soboul (Paris: Éditions sociales, 1957), p. 12.

2 Cf. Robespierre, Observations générales sur le projet d'instruction publique..., in Oeuvres Complètes, éd. sous la dir. de M. Bouloiseau, G. Lefebvre, J. Dautry, A. Soboul (Paris: PUF, 1967), Tome V, op. cit., pp. 207-11: 'L'Homme est bon, sortant des mains de la nature; quiconque nie ce principe, ne doit point songer à instituer l'homme: si l'homme est corrompu, c'est donc aux vices des institutions sociales qu'il faut imputer ce désordre. De ces deux vérités découlent tous les principes de l'éducation, soit publique, soit particulière. Si la nature a créé l'homme bon, c'est à la nature qu'il faut le ramener. Si les institutions sociales ont dépravé l'homme, ce sont les institutions sociales qu'il faut réformer.'

3 Robespierre, Oeuvres Complètes, Tome X, Discours, (5e partie: 27 juillet 1793-27 juillet 1794), op. cit., p. 353.

4 Loc. cit.

5 Saint-Just, Fragments d'Institutions républicaines, op. cit., p. 251.

6 Ibidem, p. 253.

7 Robespierre, Observations générales sur le projet d'instruction publique..., in Oeuvres Complètes, Tome V, op. cit., p. 208.

8 Ibidem.

9 Contrat, Livre II, chapitre 6: 'toute justice vient de Dieu.' 
10 Il s'agit là d'un kantisme, selon la formule de Mathiez, que Kant n'aurait sans doute pas approuvé. Nous ajouterons qu'il s'agit plutôt des règles d'une morale surtout politique et, avec elle, des principes de la religion qu'elle entraîne, c'est-à-dire $\mathrm{d}^{\prime}$ une religion civile. Par définition alors la morale et la religion robespierriste ne sont pas désintéressées, au sens où Kant l'entendrait; le citoyen fera son devoir non pas pour faire son devoir, tout court, mais pour faire des devoirs, au pluriel, lesquels sont toujours tempérés par les impératifs du politique, et de la raison publique, comme Robespierre aime à appeler de ce nom la raison d'état orientée par l'utilité générale. Robespierre, en matière de religion, lit Rousseau avec les yeux pragmatistes d'un Montesquieu, si l'on peut risquer cette image. On sait les positions de Montesquieu dont Robespierre a par ailleurs une parfaite connaissance, sur le rôle socialement utile de la religion dans l'État. Montesquieu juge des rapports entre religion et État, en politique et non en théologien, comme il dit lui-même (voir Esprit des Lois, XXIV, 1, 6, 26 notamment). En revanche, en ce qui concerne le sentiment religieux et le déisme, Robespierre est ici plus proche de Rousseau que de Montesquieu dont le refus de prendre position sur la question de l'Étre suprême est dicté surtout par l'impatience du scientifique devant la stérilité des discussions métaphysiques. Robespierre partage le théisme de Rousseau, et les conséquences sociales de cette conception, qui faisait demander au futur auteur de l'Émile et du Contrat, dans sa Lettre à Voltaire sur le désastre de Lisbonne: (18 août 1756) qu'il y eût 'dans chaque État un code moral, ou une espèce de profession de foi civile qui contînt positivement les maximes sociales que chacun serait tenu d'admettre, et négativement les maximes fanatiques qu'on serait tenu de rejetter, non comme impies, mais comme séditieuses,' cf. Rousseau, Oeuvres Complètes, Tome IV, Émile, éd. sous la dir. de B. Gagnebin et M. Raymond (Paris: Bibliothèque de la Pléiade, NRF-Gallimard, 1969), p. 1073.

11 Robespierre in Oeuvres Complètes, Tome X, Discours, (5e partie: 27 juillet 1793-27 juillet 1794), op. cit., Discours du 29 juillet 1793, p. 11. Il y reprend terme à terme le paragraphe d'ouverture du projet d'éducation de son ami Le Peletier de Saint-Fargeau qu'il présente à l'Assemblée, six mois après la mort de son auteur.

12 On peut trouver éclairant de se rapporter ici encore à la pensée du féal de Robespierre et à l'Essai de Constitution présenté par Saint-Just à la Convention, le 24 avril 1793. Les articles de la section Dispositions Fondamentales insistent conjointement au projet de Déclaration des droits de Robespierre, sur les principes fondamentaux qui s'appellent les uns les autres, savoir, la constitution d'un état n'est pas autre chose que l'application des droits et des devoirs légitimes des hommes, la souveraineté du peuple; la subordination des magistrats comme représentants du peuple à la volonté générale, le pouvoir des lois comme seul pouvoir légitime. Saint-Just, Essai de Constitution, op. cit., p. 197.

13 Voir en particulier les oeuvres de d'Holbach qui systématise les idées dominantes des Lumières philosophiques sur les rapports entre morale et politique, en particulier la Politique naturelle, et les deux Systèmes, le Système de la nature et le Système social.

14 D'Holbach, Essai sur les Préjugés, chap. II: 'La morale est fondée sur l'intérêt du genre humain [...] la politique est fondée sur les besoins de la société'; chap. VI: 'Toute morale doit se fonder sur le désir du bonheur, et pour être efficace, elle doit conduire au bonheur.' Au sujet de la vertu et de son inscription dans le domaine moral qui fait apparenter cette notion chez les Philosophes aux devoirs envers l'humanité, on trouvera, par exemple dans le Système Social, I, § 76, III, § 163, des propositions telles que les suivantes: 'La vertu est une disposition habituelle à faire ce qui contribue au bonheur des êtres de notre espèce, et à 
s'abstenir de ce qui peut leur nuire'; 'La morale est faite pour indiquer les avantages de la vertu; l'éducation doit en semer les principes; l' habitude doit en rendre la pratique familière; l'opinion publique et l'exemple doivent la soutenir; la législation doit lui donner la sanction de l'autorité; le gouvernement doit la rendre plus persuasive à l'aide des récompenses; les châtiments doivent faire trembler tous ceux que leur perversité rend sourds à la voix de la raison.'

15 D'Holbach, Système social, III, § 164.

16 Cf. les articles XI et XII du project de Déclaration de Robespierre: 'La société est obligée de pourvoir à la subsistance de tous ses membres, soit en leur procurant du travail, soit en assurant les moyens d'exister à ceux qui sont hors d'état de travailler' (article XI); 'Les secours nécessaires à l'indigence sont une dette du riche envers le pauvre: il appartient à la loi de déterminer la manière dont cette dette est acquittée' (article XII).

17 Robespierre, in Oeuvres Complètes, Tome X, Discours, (5e partie: 27 juillet 1793-27 juillet 1794), op. cit., p. 457.

18 Cf. Article V de la Déclaration des droits: 'La loi ne peut défendre que ce qui est nuisible à la société; elle ne peut ordonner que ce qui lui est utile.'

19 Cité d'après Bouloiseau, Robespierre (Paris: PUF, 1956), p. 88.

20 Marc Bouloiseau dans son Robespierre, op. cit., p. 75, rappelle à ce propos les circonstances historiques et idéologiques par lesquelles se révèlent les divergences profondes qui séparent les Robespierristes de leurs collègues Montagnards. Malgré le succès du projet présenté par Robespierre, projet qui sera imprimé sur l'ordre à la fois des Jacobins et de la Convention, celle-ci ne se ralliera pourtant qu'au texte du Comité de Salut Public, lequel bien que s'inspirant de la pensée de Robespierre en fausse son sens le plus radical. Ainsi les députés ne retiendront pas les limitations du droit de propriété; ils réduiront le principe du droit de travail pour tous à celui du recours des indigents à l'assistance publique; ils font disparaître aussi les articles ayant trait à l'impôt progressif ainsi qu'à la solidarité internationale contre la tyrannie.

21 Robespierre, Oeuvres Complètes, Tome V, op. cit., p. 208.

22 Les devoirs qui sont absents formellement du titre de la Déclaration, si on compare celle-ci à la Déclaration de 89 , réapparaissent activement de la sorte comme associés aux buts que doit servir la Déclaration.

23 On sait que ce plan démocratique prévoyait des écoles communes et gratuites, et préconisait l'instruction obligatoire et égale pour tous. Ce dernier point ne fut pas retenu par la Convention qui le jugeait contraire à l'esprit de liberté. Cf. Oeuvres Complètes, Tome X, Discours, (5e partie: 27 juillet 1793-27 juillet 1794), op. cit., pp. 11-35.

24 Ibidem, p. 35.

25 Robespierre, Oeuvres Complètes, Tome V, op. cit., p. 19.

26 A. Mathiez, le pionnier des études robespierristes, souligne dans son livre. Études sur Robespierre (Paris, c. 1958), p. 138, la réaction significative à ce propos de Robespierre dans les Notes sur les dantonistes. Robespierre qui prépare son rapport accusateur contre Danton en qui il voit un ennemi de la Révolution, s'indigne du peu de cas que fait celui-ci de la notion de vertu: 'Le mot de vertu faisait rire Danton; il n'y avait pas de vertu plus solide, disait-il plaisamment, que celle qu'il déployait toutes les nuits avec sa femme. [...] Comment un homme à qui toute idée de morale était étrangère pouvait-il être le défenseur de la liberté?' Et Robespierre note un peu plus loin: 'C'était sans doute le secret de sa politique 
qu'il révéla lui-même par un mot remarquable: ce qui rend notre cause faible, disait-il à un vrai patriote dont il feignait de partager les sentiments, c' est que la sévérité de nos principes effarouche beaucoup de monde.' Et lorsque

Robespierre, avec l'aide de Saint-Just, fera condamner Danton et ses partisans, Desmoulins, Philippaux, et d'autres au cours des procès-amalgame d'avril 1794, il espère en même temps frapper la faction de ceux qui attentent à la vertu et aux principes fondamentaux de la Montagne.

27 Cf. par exemple, Robespierre, Oeuvres Complètes, Tome V, op. cit., p. 17; Tome X, op. cit., p. 354; p. 572.

28 Robespierre, Oeuvres Complètes, Tome X, Discours, (5e partie: 27 juillet 1793-27 juillet 1794), op. cit., p. 353.

29 Ibidem.

30 Saint-Just, Esprit de la révolution et de la constitution, op. cit., quatrième partie, Ch. 1, p. 92. Saint-Just poursuit en tâchant d'établir la liberté sur le terrain politique et en affirmant que la liberté comprise autrement n'est autre que 'l'art de l'orgueil humain et c' est malheureusement en ce sens que Rousseau de Genève, tout sublime qu'il est, a toujours parlé.'

31 Saint-Just, Notes inédites, in Annales historiques de la Révolution française, oct-déc. 1949, cité d'après J. Ratinaud, Robespierre (Paris: Le Seuil, 1960), p. 179.

32 Robespierre, Oeuvres Complètes, Tome IX, Discours, (4e partie: sept. 1792-27 juillet 1793), op. cit., pp. 459-71.

33 Robespierre, Oeuvres Complètes, Tome V, op. cit., p. 19.

34 Robespierre, Oeuvres Complètes, Tome V, op. cit., p. 363.

35 Robespierre, Oeuvres Complètes, Tome V, op. cit., p. 209.

36 Cf. Rousseau, le Contrat social, op. cit., livre II, chap. VII.

37 Cf. Robespierre in Oeuvres Complètes, Tome V, op. cit., p. 19: 'Il faudrait une intelligence supérieure qui vît toutes les passions et qui n'en éprouvât aucune, qui, dans le progrès des tems, se ménageant une gloire éloignée, pût travailler dans un siècle, et jouir dans un autre. Il faudroit des Dieux pour donner des lois aux hommes. Il faut au moins des philosophes également éclairés et intrépides, qui éprouvent les passions des hommes; mais dont la première passion soit l'horreur de la tyrannie et l'amour de l'humanité; foulant aux pieds la vanité, l'envie, l'ambition et toutes les foiblesses des petites âmes, inexorables pour le crime armeé du pouvoir, indulgens pour l'erreur, compatissans pour la misère, tendres et respectueux pour le peuple. La première chose que doit savoir le législateur, c'est que le peuple est bon: le premier sentiment qu'il doit éprouver c'est le besoin de venger ses injures et de lui rendre toute sa dignité.'

38 Robespierre, 'Projet...' in Oeuvres Complètes, Tome V, op. cit., pp. 361, article IV; vr. aussi pour le texte complet du projet de la Déclaration, Oeuvres Complètes, Tome IX, Discours, (4e partie: sept. 1792-27 juillet 1793), op. cit., discours du 21 avril aux Jacobins, pp. 454-56 et du 24 avril devant la Convention, pp. 459-71.

39 J.-J. Rousseau, Contrat Social, op. cit., livre IV, chap. VIII.

40 L'article IV de la Déclaration qui définit la liberté continue en énonçant sa forme politique, le droit d'assemblée, la liberté d'expression, etc., en ayant soin de souligner que la nécessité de formuler ces droits qui ne sont que l'exercise normal de la liberté, suppose par là même ou la présence ou le souvenir récent du despotisme.

41 Rousseau, Contrat, op. cit., I, 4. 
42 Robespierre, Oeuvres complètes, Tome VI, Discours, (1re partie: 1789-1790), op. cit., pp. 39-70.

43 Ibidem, p. 126.

44 Cf. Robespierre, Oeuvres Complètes, Tome V, op. cit., pp. 57-58. Voir aussi Oeuvres Complètes, Tome IX, Discours, (4e partie: sept. 1792-27 juillet 1793), op. cit., Discours du 3 déc 1792, p. 123.

45 Robespierre, Oeuvres complètes, Tome VI, Discours, (1re partie: 1789-1790), op. cit., p. 88.

46 Rousseau, Contrat, op. cit., I, 8.

47 Saint-Just, in Esprit de la Révolution et de la Constitution, op. cit., chap. 3, pp. 54-55.

48 Saint-Just, in Oeuvres Complètes, édition de C. Vellay, Paris, Charpentier et Fasquelle, 2 volumes, 1908, 'Rapport sur la loi contre les Anglais' (16 octobre 1793), tome 2, p. 100: 'Nous devons donc rester continuellement en état d'énergie afin de briser également et les pièges connus et les pièges cachés.'

49 Robespierre, Oeuvres Complètes, Tome X, Discours, (5e partie: 27 juillet 1793-27 juillet 1794), op. cit., Sur les principes de morale politique..., p. 352. 